\title{
Large cell neuroendocrine carcinoma of the lung: chemotherapy regimen depends on how "large" your diagnostic criteria are
}

\author{
To the Editor:
}

We read with great interest the article by Derks et al. [1] on the efficacy of different chemotherapeutic regimens in a large series of 128 patients with stage IV large cell neuroendocrine carcinoma (LCNEC) of the lung. As outlined by the authors, in 2005 , we dealt with the same dilemma when analysing 83 surgically resected LCNECs collected at two different institutions [2]. In our study, patients with metastatic disease treated either in an adjuvant setting or post-surgery with platinum/etoposide, a conventional regimen for small cell lung cancer (SCLC), showed a significant clinical benefit in terms of overall survival compared to patients treated with chemotherapeutic regimens used for non-small cell lung cancer (NSCLC). In contrast, DeRKS et al. [1] showed that patients with metastatic LCNEC receiving platinum/ gemcitabine, a regimen commonly used in NSCLC, displayed a longer overall survival than those receiving SCLC-oriented chemotherapy. In our view, a number of reasons, discussed herein, may account for the apparent discrepancies between these and other studies [3]. In our study [2], LCNEC cases eventually represented less than one-quarter $(83(23.8 \%)$ out of 348$)$ of all lung cancer cases identified based on morphological examination alone. In addition, although we applied strict diagnostic criteria, some cases required a consensus diagnosis among all three thoracic pathologists involved in the histological review, highlighting the difficulties encountered when making a diagnosis of LCNEC, even amongst expert pathologists [4]. In agreement with the last two World Health Organization (WHO) classifications of lung tumours [5] suggesting to limit/avoid the diagnosis of LCNEC on cytology/small biopsy, we included only surgically resected cases (i.e. none of our original cases was in stage IV at the time of the diagnosis). Conversely, DerKs et al. [1] and another recent study by NAIDOo et al. [6] included only stage IV LCNEC cases diagnosed on biopsy.

Thus, it is impossible to compare the results of studies that, while formally focusing on LCNEC, looked at different tumour stage and possibly different histology.

DERKS et al. [7] have recently proposed to introduce neuroendocrine markers along with thyroid transcription factor 1 and p40 into the diagnostic panel aimed at identifying LCNEC or possible LCNEC among poorly differentiated NSCLC. In their reply, TRAVIS et al. [8] reinforced the concept that neuroendocrine markers should be used only in cases overtly showing features indicative of neuroendocrine differentiation on morphological grounds, according to the WHO scheme [5].

An extensive and often controversial use of immunostains when dealing with poorly differentiated NSCLC could account for the increased frequency of LCNEC. Indeed, it is well established that an "aberrant" expression of neuroendocrine markers in overt NSCLC may be observed in up to $30 \%$ of cases. This phenomenon is not surprising. In fact, in their seminal study, BHATTACHARJeE et al. [9] identified a subset of adenocarcinomas with neuroendocrine differentiation associated with a significantly worse prognosis, highlighting an important overlap between LCNEC and adenocarcinoma even at the gene expression profiling level. Taking all these data into account, it is intuitive that the bar at which pathologists make a diagnosis of LCNEC is still inconsistent. In other words, LCNEC remains a difficult and somewhat subjective diagnosis reflecting the differences in interpretation and use of morphological features and/or immunostains, respectively. In support of this view, molecular investigations in LCNEC have recently revealed that what pathologists identify as LCNEC on morphological and immunohistochemical levels rather represents a spectrum of heterogeneous tumours harbouring different gene alterations. Using a

@ERSpublications

Diagnostic criteria influence the correct chemotherapy in LCNEC http://ow.ly/MSPR30ePZNK

Cite this article as: Rossi G, Longo L, Barbieri F, et al. Large cell neuroendocrine carcinoma of the lung: chemotherapy regimen depends on how "large" your diagnostic criteria are. Eur Respir J 2017; 50: 1701292 [https://doi.org/10.1183/13993003.01292-2017]. 


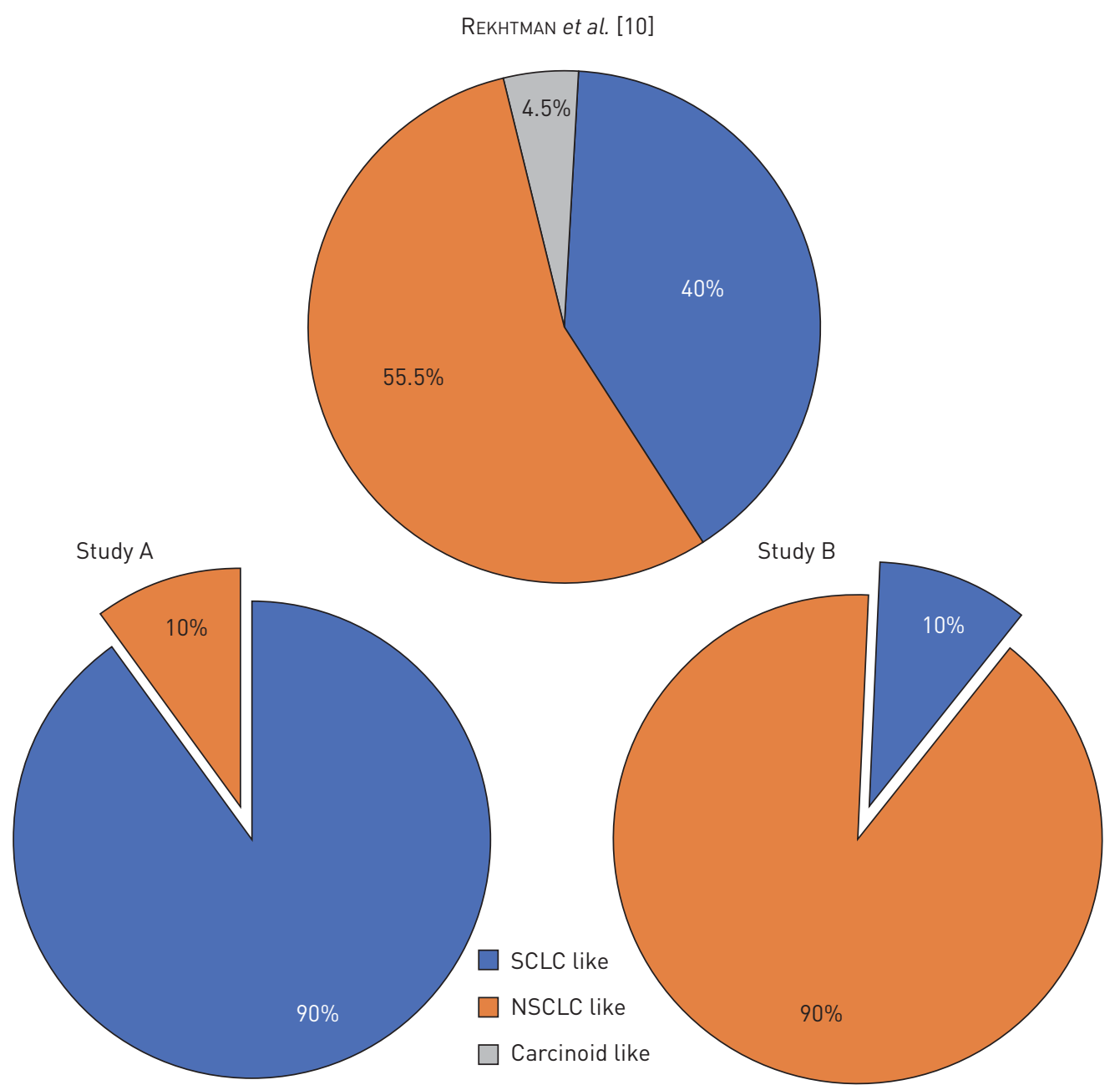

FIGURE 1 Hypothetical situation of clinical-pathological studies of large cell neuroendocrine carcinoma (LCNEC) including different rates of tumours with prevalent small cell lung cancer (SCLC)-based (study A), non-small cell lung cancer (NSCLC)-based (study B) or mixed [10] genotypes.

next-generation sequencing approach, REKHTMAN et al. [10] demonstrated that $40 \%$ of LCNECs are close to SCLC (TP53 and RB1 gene alterations), while 55.5\% are more akin to NSCLC and particularly adenocarcinomas (STK11, KRAS and KEAP1 mutations), and 4.5\% has a carcinoid-like molecular genotype (MEN1 mutations). Surprisingly, NAIDOo et al. [6] identified KRAS mutations in 24\% of stage IV LCNEC cases diagnosed on biopsy, a figure closer to that of adenocarcinoma than to SCLC. Considering this amount of molecular data, we are even more convinced that current criteria to define LCNEC are far from being perfect and studies on this topic either included a large number of SCLC-like LCNEC or collected a prevalent quota of NSCLC-like LCNEC, which in turn has created major controversies when it comes to the efficacy of different chemotherapy regimens (figure 1).

Major issues, outlined here, need to be resolved if we are to improve our knowledge of this in-between tumour entity. In this regard, we call for a consensus definition of LCNEC based on more stringent criteria, such as a correct integration of morphology and immunostains, as well as tools that allow exclusion (e.g. KRAS, EGFR and LKB1) or confirmation (e.g. TP53 and RB1) of gene alterations, particularly when dealing with cytology and small biopsies. Last but not least, a large, prospective multicentre clinical trial of LCNEC with centralised review of the diagnosis and use of genetic investigations is urgently needed.

Giulio Rossi ${ }^{1}$, Lucia Longo ${ }^{2}$, Fausto Barbieri ${ }^{3}$, Federica Bertolini ${ }^{3}$ and Paolo Spagnolo ${ }^{4}$

${ }^{1}$ Operative Unit of Pathologic Anatomy, Azienda USL, Valle d'Aosta, Aosta, Italy. ${ }^{2}$ Oncology Unit, Hospital "Ramazzini", AUSL Modena, Carpi, Italy. ${ }^{3}$ Operative Unit of Oncology, Azienda Ospedaliero-Universitaria Policlinico, Modena, Italy. ${ }^{4}$ Section of Respiratory Diseases, Dept of Cardiac, Thoracic and Vascular Sciences, University of Padua, Padua, Italy. 
Correspondence: Federica Bertolini, Operative Unit of Oncology, Azienda Ospedaliero-Universitaria Policlinico, via del Pozzo 71, 41124 Modena, Italy. E-mail: federica.bertolini@tiscali.it

Received: June 272017 | Accepted: Aug 072017

Conflict of interest: None declared

\section{References}

1 Derks JL, van Suylen RJ, Thunnissen E, et al. Chemotherapy for pulmonary large cell neuroendocrine carcinomas: does the regimen matter? Eur Respir J 2017; 49: 1601838.

2 Rossi G, Cavazza A, Marchioni A, et al. Role of chemotherapy and the receptor tyrosine kinases KIT, PDGFRa, PDGFRb, and Met in large-cell neuroendocrine carcinoma of the lung. J Clin Oncol 2005; 23: 8774-8785.

3 Prelaj A, Rebuzzi SE, Del Bene G, et al. Evaluation of the efficacy of cisplatin-etoposide and the role of thoracic radiotherapy and prophylactic cranial irradiation in LCNEC. ERJ Open Res 2017; 3: 00128-2016.

4 Travis WD, Gal AA, Colby TV, et al. Reproducibility of neuroendocrine lung tumor classification. Hum Pathol 1998; 29: 272-279.

5 Travis WD, Brambilla E, Burke A, et al., eds. WHO Classification of Tumours of the Lung, Pleura, Thymus and Heart. Lyon, IARC Press, 2015.

6 Naidoo J, Santos-Zabala ML, Iyriboz T, et al. Large cell neuroendocrine carcinoma of the lung: clinico-pathologic features, treatment, and outcomes. Clin Lung Cancer 2016; 17: e121-e129.

7 Derks JL, Speel EJ, Dingemans AM. An unmet need in the WHO 2015 biopsy classification: poorly differentiated NSCCs with positive neuroendocrine markers. J Thorac Oncol 2016; 11: e25-e26.

8 Travis WD, Brambilla E, Nicholson AG. Testing for neuroendocrine immunohistochemical markers should not be performed in poorly differentiated NSCCs in the absence of neuroendocrine morphologic features according to the 2015 WHO classification. J Thorac Oncol 2016; 11: e26-e27.

9 Bhattacharjee A, Richards WG, Staunton J, et al. Classification of human lung carcinomas by mRNA expression profiling reveals distinct adenocarcinoma subclasses. Proc Natl Acad Sci USA 2001; 98: 13790-13795.

10 Rekhtman N, Pietanza MC, Hellmann M, et al. Next-generation sequencing of pulmonary large cell neuroendocrine carcinoma reveals small cell carcinoma-like, and non-small cell carcinoma-like subsets. Clin Cancer Res 2016; 22: 3618-3629.

\section{Why we should improve current practice of diagnosing and treating pulmonary large cell neuroendocrine carcinomas in patients with advanced disease}

From the authors:

In response to our manuscript on first-line chemotherapy treatment for metastatic pulmonary large cell neuroendocrine carcinoma (LCNEC) [1], Rossi and co-workers raised their concerns about the validity of our results by questioning the accuracy of LCNEC diagnosis on a biopsy specimen. We would like to thank the authors for their critical appraisal, underscoring the need to increase awareness among pulmonologists, oncologists and pathologists of the diagnostic issues and consequences of metastatic LCNEC diagnosed on a biopsy specimen.

The diagnostic criteria for LCNEC according to the current World Health Organization (WHO) classification (2015) include a high number of mitoses ( $>10$ mitoses per $2 \mathrm{~mm}^{2}$ ), neuroendocrine morphology such as rosettes, trabecular growth pattern or palisading of cells, and neuroendocrine differentiation identified by immunohistochemical markers or electron microscopy [2]. Although the

@ERSpublications

Current criteria for LCNEC diagnosed on a biopsy specimen are in need of improvement http://ow.ly/lRP730fu552

Cite this article as: Derks J, van Suylen RJ, Thunnissen E, et al. Why we should improve current practice of diagnosing and treating pulmonary large cell neuroendocrine carcinomas in patients with advanced disease. Eur Respir J 2017; 50: 1701658 [https://doi.org/10.1183/13993003.01658-2017]. 
current classification provides tools to diagnose lung cancer on small biopsies, this is not the case for LCNEC. Nevertheless a diagnosis of "nonsmall cell lung carcinoma (NSCLC) favouring LCNEC" can be made if the above criteria are identified in a biopsy specimen.

A comparison of pre-operative biopsy with the surgical resection specimen from identical anatomic locations including carcinoids and LCNEC/small cell lung cancer (SCLC), demonstrated that a Ki-67 cut-off of $>20 \%$ is specific and sensitive on a biopsy specimen to separate carcinoid from high-grade neuroendocrine carcinomas [3]. We recently proposed introduction of neuroendocrine markers along with thyroid transcription factor-1 and p40 into the diagnostic panel aiming to increase the yield of LCNEC diagnoses on biopsies [4]. This is important because in pre-operatively obtained biopsy specimens of surgically confirmed LCNEC, neuroendocrine morphology may be difficult to recognise leading to a diagnosis of NSCLC-not otherwise specified (NOS) or favouring adenocarcinoma diagnosis. The application of additional neuroendocrine markers and use of core needle biopsies may solve these diagnostic issues in LCNEC ( J. Derks and co-workers; unpublished data).

In our study on the treatment of metastatic LCNEC, three expert thoracic pathologists reviewed 207 cases that were diagnosed as LCNEC between 2003 and 2012 [1]. Eventually, 62\% were confirmed as LCNEC, other diagnoses included were (atypical) carcinoid, SCLC and NSCLC with neuroendocrine differentiation. Since LCNEC has only been officially recognised by the WHO since 1999, it is understandable that the percentage of LCNEC identified in our study is higher than that in the work of Rossi et al. [5], who studied LCNEC from 1990 to 2004. In previous work, we reported that LCNEC is diagnosed with an increased frequency on biopsy specimens [6]. Thus, although the WHO 2015 classification may not promote the diagnosis of LCNEC on a biopsy specimen, this frequently occurs in daily practice, putting clinicians on the spot how to treat such patients.

The aim of our study was to evaluate different chemotherapy treatments in de novo metastatic LCNEC in current clinical practice. Our results indicate that LCNEC should not be routinely treated as SCLC. In addition to the results obtained in our study, this is also supported by a phase II trial showing favourable effects of carboplatin-paclitaxel chemotherapy followed by maintenance everolimus (mTOR inhibitor) [7]. Although the study of Rossi et al. [5] only included surgical resection specimens, the results may be biased as they compared only a few LCNEC patients $(n=27)$ part of whom were treated with radiotherapy and/or without a platinum compound in the NSCLC treatment arm. Furthermore, the median survival rates observed for metastatic LCNEC in the study of Rossi et al. (i.e. 51 months in the platinum-etoposide arm versus \pm 7 months reported by a phase II study) seems unusually high and may suggest patient selection [8].

In their correspondence, Rossi and co-workers referred to molecular findings in LCNEC published by REKHTMAN et al. [9] ( $\mathrm{n}=45$ LCNEC). Previously, similar data were also presented by GeORGE et al. [10] $(\mathrm{n}=75)$. Both studies proposed that LCNEC should be subdivided into at least two subtypes, i.e. a TP53-RB1 mutated subtype (SCLC like) and LCNEC exhibiting a mutation in STK11, KEAP1 or KRAS with wild-type RB1 (NSCLC like). It is tempting to speculate that these molecular subgroups relate to chemotherapy outcomes in LCNEC. In this context, at the American Society of Clinical Oncology 2017 meeting we presented our analysis of the, in part, current cohort of LCNEC (published in the European Respiratory Journal $(E R J)$ ) for these molecular subtypes using next-generation sequencing [11]. This analysis indeed suggests that the treatment outcomes of metastatic LCNEC may be influenced by different molecular subtypes. Patients having LCNEC with RB1 wild-type (and/or STK11 mutations, i.e. "NSCLC like") had longer overall and progression-free survival when treated with platinum-gemcitabine or taxane chemotherapy versus platinum-etoposide chemotherapy.

In summary, we support the call for a consensus definition from Rossi and co-workers. The WHO 2015 criteria for LCNEC diagnosed on a biopsy specimen are in need of improvement and should include, amongst others, molecular analysis of LCNEC validated within a large multicentre clinical trial. Nevertheless, until such studies have been performed, the work we presented in the ERJ represents the largest comparison of first-line chemotherapy treatment in carefully pathology revised confirmed metastatic LCNEC. Taken together, when it comes to studying LCNEC "size does matter" but it is likely that in the near future a "small (biopsy) size fits all".

Jules Derks $^{1}$, Robert Jan van Suylen ${ }^{2}$, Erik Thunnissen ${ }^{3}$, Michael den Bakker ${ }^{4,5}$, Harry Groen ${ }^{6}$, Egbert Smit ${ }^{7,8}$, Ronald Damhuis ${ }^{9}$, Esther van den Broek ${ }^{10}$, Ernst-Jan Speel ${ }^{11}$ and Anne-Marie C. Dingemans ${ }^{1}$

${ }^{1}$ Dept of Pulmonary Diseases, GROW school for Oncology and Developmental Biology, Maastricht University Medical Centre, Maastricht, The Netherlands. ${ }^{2}$ Pathology-DNA, location Jeroen Bosch Hospital, s' Hertogenbosch, The Netherlands. ${ }^{3}$ Dept of Pathology, VU University Medical Centre, Amsterdam, The Netherlands. ${ }^{4}$ Dept of Pathology, Maasstad hospital, Rotterdam, The Netherlands. ${ }^{5}$ Dept of Pathology, Erasmus MC, Rotterdam, The Netherlands. ${ }^{6}$ Dept of Pulmonary Diseases, University of Groningen and University Medical Centre, Groningen, The Netherlands. ${ }^{7}$ Dept of Pulmonary Diseases, VU medical center, Amsterdam, The Netherlands. ${ }^{8}$ Dept of Thoracic Oncology, Netherlands Cancer Institute, Amsterdam, The Netherlands. ${ }^{9}$ Department Research, Comprehensive Cancer Association, Utrecht, 
The Netherlands. ${ }^{10}$ PALGA Foundation, Houten, The Netherlands. ${ }^{11}$ Dept of Pathology, GROW school for Oncology and Developmental Biology, Maastricht University Medical Centre, Maastricht, The Netherlands.

Correspondence: Anne-Marie. C. Dingemans, Dept of Pulmonology, P. Debyelaan 25, Postbox 58006202 AZ, Maastricht, The Netherlands. E-mail: a.dingemans@mumc.nl

Received: Aug 162017 | Accepted: Sept 162017

Conflict of interest: None declared.

\section{References}

1 Derks JL, van Suylen RJ, Thunnissen E, et al. Chemotherapy for pulmonary large cell neuroendocrine carcinomas: does the regimen matter? Eur Respir J 2017; 49: 1601838

2 Travis WD, Brambilla E, Burke AP, et al. Introduction to the 2015 World Health Organization classification of tumors of the lung, pleura, thymus, and heart. J Thorac Oncol 2015; 10: 1240-1242.

3 Fabbri A, Cossa M, Sonzogni A, et al. Ki-67 labeling index of neuroendocrine tumors of the lung has a high level of correspondence between biopsy samples and surgical specimens when strict counting guidelines are applied. Virchows Arch 2017; 470: 153-164.

4 Derks JL, Speel EJ, Dingemans AM. An unmet need in the WHO 2015 biopsy classification: poorly differentiated NSCCs with positive neuroendocrine markers. J Thorac Oncol 2016; 11: e25-e26.

5 Rossi G, Cavazza A, Marchioni A, et al. Role of chemotherapy and the receptor tyrosine kinases KIT, PDGFR $\alpha$, PDGFR $\beta$, and Met in large-cell neuroendocrine carcinoma of the lung. J Clin Oncol 2005; 23: 8774-8785.

6 Derks JL, van Suylen RJ, Thunnissen E, et al. A population-based analysis of application of WHO nomenclature in pathology reports of pulmonary neuroendocrine tumors. J Thorac Oncol 2016; 11: 593-602.

7 Christopoulos P, Engel-Riedel W, Grohé C, et al. Everolimus with paclitaxel and carboplatin as first-line treatment for metastatic large-cell neuroendocrine lung carcinoma: a multicenter phase II trial. Ann Oncol 2017; 28: 1898-1902.

8 Le Treut J, Sault MC, Lena H, et al. Multicentre phase II study of cisplatin-etoposide chemotherapy for advanced large-cell neuroendocrine lung carcinoma: the GFPC 0302 study. Ann Oncol 2013; 24: 1548-1552.

9 Rekhtman N, Pietanza MC, Hellmann M, et al. Next-generation sequencing of pulmonary large cell neuroendocrine carcinoma reveals small cell carcinoma-like and non-small cell carcinoma-like subsets. Clin Cancer Res 2016; 22: 3618-3629.

10 George J, Fernandez-Cuesta L, Vonn W, et al. Comparative analysis of small cell lung cancer and other pulmonary neuroendocrine tumors. AACR Cancer Res 2016; 76: Suppl., Abstract nr 122.

11 Derks J, Leblay N, van Suylen RJ, et al. Genetic subtypes of large cell neuroendocrine carcinoma (LCNEC) to predict response to chemotherapy. J Clin Oncol 2017; 35: Suppl., 9061-9061. 